\title{
Accuracy of Baseline and Complex Methods Applied to Morphosyntactic Tagging of Polish
}

\author{
Marcin Kuta, Michał Wrzeszcz, Paweł Chrząszcz, and Jacek Kitowski \\ Institute of Computer Science, AGH-UST, al. Mickiewicza 30, Kraków, Poland \\ \{mkuta,kito\}@agh.edu.pl
}

\begin{abstract}
The paper presents baseline and complex part-of-speech taggers applied to the modified corpus of Frequency Dictionary of Contemporary Polish. Accuracy of 5 baseline part-of-speech taggers is reported. On the base of these results complex methods are worked out. Thematic split and attribute split methods are proposed and evaluated. Tagging accuracy of voting methods is evaluated finally. The most accurate baseline taggers are SVMTool (for a simple tagset) and fnTBL (for a complex tagset). Voting method called Total Precision achieves the top accuracy among all looked over methods.
\end{abstract}

Keywords: part-of-speech tagging, natural language processing.

\section{Introduction}

Part-of-speech (POS) tagging algorithms are intensively exploited in a wide range of applications including syntactic and semantic parsing, speech recognition and generation, ontology construction, machine translation, text understanding, information retrieval and many others [1].

Unfortunately POS tagging of highly inflecting languages like Polish is much more challenging than application to analytic languages (e.g. English or French), as the former are annotated with large, complex tagsets, describing many morphological categories.

POS tagging algorithms are computationally time demanding, especially when employed to inflecting languages. In the domain training time exceeding $24 \mathrm{~h}$ is nothing extraordinary. Time requirements of the complex algorithms like split models or voting methods are moreover one order of magnitude higher.

The paper examines accuracy of selected baseline algorithms applied to morphosyntactic tagging of Polish. Next more complex methods are investigated, both originally proposed (split models) as well as already known but not evaluated on Polish (voting methods). We present also results for the simple tagset (only the first attribute of each tag considered) to provide point of reference to English and other languages described by small tagsets.

The taggers are evaluated on the modified corpus of Frequency Dictionary of Contemporary Polish (m-FDCP), authors' improved version [2] of the standard FDCP corpus available at [3] site. The m-FDCP corpus is annotated with the complex tagset, containing over 1200 tags. Tags consist of a set of attributes, 
each attribute describing selected morphological category. A token is the entity being subject of tagging. A word segment is a token containing at least one letter or digit. By raw text we mean a sequence of tokens without their tags.

\section{Baseline Tagging Algorithms}

POS tagging algorithms are roughly divided into statistical and rule based methods. For a given token $w$ all methods examine its context, being a window of $N$ tokens and tags centred on the token $w$. The rule based methods take into account a wide context, which is desirable in the case of languages containing long-distance syntax dependencies. Statistical algorithms map a sequence of tokens into a sequence of tags with a probability model, which describes occurrence of the most probable sequence of tags for a given sequence of tokens.

\subsection{Evaluated Algorithms}

In the paper we first investigate five baseline algorithms, providing new results, compared to [2] 4]. The methods serve furthermore as components for construction of more sophisticated taggers.

Hidden Markov Model (HMM). The algorithm belongs to the statistical methods. Given a sequence of tokens, $w_{1}, \ldots, w_{n}$, the HMM tagger assigns a sequence of tags, $T=\left(t_{1}, \ldots, t_{n}\right)$, according to a formula

$$
\hat{T}=\underset{T}{\arg \max } \prod_{i}^{n} p\left(w_{i} \mid t_{i}\right) \cdot p\left(t_{i} \mid t_{i-1}, \ldots, t_{i-N}\right),
$$

where $p\left(w_{i} \mid t_{i}\right)$ is the conditional probability of occurrence of word $w_{i}$ given that tag $t_{i}$ occurred and $p\left(t_{i} \mid t_{i-1}, \ldots, t_{i-N}\right)$ is the conditional probability of occurrence of tag $t_{i}$ given that tag sequence $t_{i-1}, \ldots, t_{i-N}$ previously occurred.

Maximum entropy. This statistical method [5] aims to maximize the entropy function by selection of binary features, reflecting dependence in a training corpus. The model assumes a set of binary features, $f_{j}$, is defined on the combination of a tag $t_{i}$ and its context $c$. The probabilistic model is built from family of models

$$
p\left(t_{i}, c\right)=\pi \mu \prod_{j} \alpha_{j}^{f_{j}\left(t_{i}, c\right)},
$$

where $p\left(t_{i}, c\right)$ stands for joint distribution of tags and contexts and $\pi, \mu$ are normalisation factors in order that $p(\cdot, \cdot)$ forms the probability function.

Memory-based learning. The algorithm exploits rule based approach. Tagger 6] acquires examples from training corpora, which are later used in the tagging process. During the learning process memory-based taggers store in memory a set of examples $\left(t_{i}, c_{i}\right)$, where $t_{i}$ denotes the tag and $c_{i}$ its context. Given a token 
$w$ in context $c$, the memory-based tagger assigns it a tag $t_{k}$, such that distance between $c$ and $c_{k}$ is minimal.

Transformation-based error-driven learning. This rule based method [7] starts with assigning a trivial sequence of tags to a given tokenised text. The target sequence of tags is determined by applying series of transformations. Each transformation, $F$, is a rule in the form: "Replace value of tag $t$ with value $y$ if current context $c$ fulfils condition $\phi . "$ The core of learning process is the algorithm for finding suitable transformations.

Support vector machines. The SVM is a statistical algorithm, which maps input data to a higher dimensional space and next constructs a linear separating hyperplane with the maximal margin. The mapping is done with kernel functions, of which linear, polynomial, radial basis (RBFs) and sigmoid functions are the most used. The SVM approach achieves $97.16 \%$ accuracy for English [8].

As an implementation of the above algorithms the following taggers have been chosen for evaluation:

Table 1. Taggers used in experiments

\begin{tabular}{|c|c|}
\hline Algorithm & Tagger name \\
\hline$\overline{\mathrm{HMM}}$ & TnT [9] \\
\hline Maximum entropy & MXPost $^{1}$ [5] \\
\hline Transformation based & fnTBL [10] \\
\hline Memory based & MBT [6] \\
\hline SVM & SVMTool $^{2}[8$ \\
\hline
\end{tabular}

Next the complex methods have been worked out: the split models have been elaborated and the collective methods evaluated.

\section{$3 \quad$ Split Models}

\subsection{Thematic Split}

To make benefit of the thematic split approach the corpus structure should be nonuniform, i.e., it should consist of segments diversified in language style. This is the case of the m-FDCP corpus, containing 5 segments (thematic parts) differing in vocabulary, style, etc., e.g. average sentence length varies from 10.42 tokens/sentence (artistic drama) to 23.27 tokens/sentence (popular science).

These differences cause that tagging rules utile in one segment may become inefficient for another one. Thus, instead of providing one overall language model, created from the whole corpus, it is worth considering building a number of separate models, each acquired with a baseline tagger from a different thematic part.

\footnotetext{
${ }^{1}$ Referred further as MXP.

${ }^{2}$ Referred further as SVM.
} 


\subsection{Attribute Split}

Attribute split method is applicable only to corpora annotated with complex tagsets. Assuming a tagset provides for presence of $K$ morphological categories, the entire corpus is replicated $K$ times, each copy corresponding to one morphological category. The $i$-th copy $(1 \leq i \leq K)$ contains the whole text (all tokens), annotated with a small tagset $\mathcal{T}_{i}$. The tagset $\mathcal{T}_{i}$ is obtained from the complex tagset by removing from each tag all attributes except the attribute describing $i$-th morphological category. If $i$-th morphological category is not applicable to a current token, the token is annotated with a special tag none in a relevant copy.

Next, the training procedure and tagging of the raw test set is performed separately on each copy with a given baseline algorithm. Partition to training and test sets remains preserved as in the original corpus annotated with the complex tagset. Finally, $K$ output files, generated within tagging of the raw test set, are merged into one file, where each token is back annotated with all the relevant morphological categories. The merged file is evaluated against the test set of the corpus annotated with the complex tagset.

\section{Collective Methods}

Collective methods and their performance on English and Dutch are shown in the fundamental work [11. The idea of collective methods is based on assumption, that different baseline methods make errors in different places, i.e., baseline methods are to some extent complementary. The higher the complementarity of taggers, the bigger chance the combined system compensates errors of its constituents and performs better than the components alone. A few independent baseline taggers (components) propose a tag simultaneously [11] [12] [13] according to their algorithms described in Sect. 2.1. Proposed tags are compared and the best tag is selected according to an arbitration mechanism. As an arbitration several voting strategies are available.

Assuming the reference test corpus contains $n$ tokens $w_{1}, \ldots, w_{n}$, a token $w_{i}$ is annotated in the corpus with a tag $t_{i}(1 \leq i \leq n)$, a tagger $A$ guesses for a token $w_{i}$ a tag $t_{i}^{A}$, the accuracy of the tagger $A$ is defined as follows:

$$
\text { accuracy } \stackrel{\text { df }}{=} \frac{\# \text { correctly tagged tokens }}{\# \text { all tokens }}=\frac{\sum_{i=1}^{n} \delta\left(t_{i}^{A}, t_{i}\right)}{n},
$$

where $\delta$ is the Kronecker delta function. The sentence accuracy is a ratio of entirely correctly tagged sentences to the total number of sentences.

If a tagger $B$ guesses a tag $t_{i}^{B}$ respectively, the complementarity of the tagger $B$ to the tagger $A, \operatorname{comp}(B \mid A)$, is determined as follows [14]:

$$
\operatorname{comp}(B \mid A) \stackrel{\mathrm{df}}{=} 1-\frac{\# \text { common errors of taggers } A \text { and } B}{\# \text { errors of tagger } A} .
$$

Given a tag $X$, the precision and recall of the tagger $A$ on the $\operatorname{tag} X$ are given as:

$$
\operatorname{prec}_{X} \stackrel{\text { df }}{=} \frac{\# \text { tokens tagged and annotated with } X}{\# \text { tokens tagged with } X},
$$




$$
\text { recall }_{X} \stackrel{\text { df }}{=} \frac{\text { ttokens tagged and annotated with } X}{\# \text { tokens annotated with } X} .
$$

Majority is a simple voting method, with exactly one vote assigned to each tagger. With weighted voting methods each tagger votes for its accuracy (Total Precision method) or $\operatorname{prec}_{X}$ (Tag Precision or Precision-Recall method). Additionally, the tagger may be obliged to support tags other than suggested by itself (Precision-Recall method, with weight $1-$ recall $_{Y}$ ). Ties are resolved by a random selection amongst winning tags. Vote strength of particular methods is summarised in Table 2 .

Table 2. Vote strength of a component tagger in various voting methods, $X$ stands for a tag proposed by tagger, $Y(Y \neq X)$ represents each tag proposed by opposition

\begin{tabular}{|l|c|c|}
\hline Voting method & $\operatorname{Tag} X$ & $\operatorname{Tag} Y$ \\
\hline Majority & 1 & 0 \\
Total Precision & accuracy & 0 \\
Tag Precision & prec $_{X}$ & 0 \\
Precision-Recall & prec $_{X}$ & $1-$ recall $_{Y}$ \\
\hline
\end{tabular}

For each tagger parameters accuracy, prec $_{X}$ and recall $_{X}$ (for each tag $X$ ) are to be determined with help of a tuning set, disjoint from a test set, to avoid artificial boosting of the above methods.

\section{Evaluated Data and Experiments Setup}

We used the modified corpus of Frequency Dictionary of Contemporary Polish [15], annotated with the slightly abridged version of the IPI PAN tagset [16]. The m-FDCP corpus is partially corrected and disambiguated version of the FDCP corpus 3, both with manual checking and automatic procedures. The whole process of corpus improving has been described in details in [2].

The corpus is balanced between five thematic parts: (A) popular science, (B) news dispatches, (C) editorials and longer articles, (D) artistic prose and (E) artistic drama, each standing for approximately $20 \%$ of the corpus and representing different style of the language. The used tagset provides for 9 morphological categories: grammatical class (part of speech or POS), number, case, gender, person, degree, aspect, negation, vocalicity. The main parameters of the corpus are gathered in Table 3 (4th column).

The baseline algorithms have been evaluated with the split of the m-FDCP corpus to a training and test set, standing for $90 \%$ and $10 \%$ of the corpus, respectively. The balanced character of the training and test set was carefully preserved within the split. Their characteristics are gathered in Table 3 ,

The setup for the thematic split approach was the following: for each of 5 thematic parts of the m-FDCP corpus the separate experiment with the baseline 
Table 3. Main parameters of the m-FDCP corpus [2]

\begin{tabular}{|l|r|r|r|}
\hline & $\begin{array}{c}\text { Training } \\
90 \%\end{array}$ & $\begin{array}{r}\text { Test } \\
10 \%\end{array}$ & \multicolumn{1}{c|}{ Full } \\
& 592729 & 65927 & 658656 \\
\hline tokens & 496907 & 55139 & 552046 \\
word segments & 36601 & 4211 & 40812 \\
sentences & 87097 & 19557 & 92872 \\
different tokens & 30 & 30 & 30 \\
\hline \multicolumn{4}{|c|}{ Simple tagset } \\
\hline tagset size 36.15 & 26.19 & 26.16 \\
ambiguous tokens, \% & 1.44 & 1.43 & 1.44 \\
mean token ambiguity & 1191 & 724 & 1243 \\
\hline \multicolumn{3}{|c|}{ Complex tagset } \\
\hline tagset size & 47.76 & 47.65 & 47.74 \\
ambiguous tokens, \% & 3.12 & 3.12 & 3.12 \\
mean token ambiguity
\end{tabular}

taggers was performed. Each part was split to a training and test set in $90 \% / 10 \%$ ratio ( $18 \%$ and $2 \%$ of the whole corpus respectively).

In the attribute split approach we used the entire m-FDCP corpus, divided to training and test sets as for baseline tagging. This corpus was replicated 9 times, each with an appropriate tagset, as there are 9 morphological categories.

The setup for voting methods was slightly more complex. We avoided partition of the corpus to training, tuning and test sets in $80 \% / 10 \% / 10 \%$ ratio [12] but instead the tuning set was created on the base of the $90 \%$ training set, used already within baseline tagging experiments. The $90 \%$ training set was divided into 9 equal parts among which 8 parts served for training and one part for testing. The procedure was repeated 9 times, with a different part serving for testing each time. The 9 output files were merged into one file, standing for the tuning set; cf. [11].

In this way two important aims have been achieved. We got bigger training set (90\% instead of $80 \%$ of the corpus) for training the baseline taggers and at the same time 9 times bigger tuning set (90\% instead of $10 \%$ of the corpus).

As baseline components we used the taggers from Table 1, prepared already for the baseline experiments. But when applied to the complex tagset, voting methods omitted the SVM tagger, as achieving much lower accuracy than the rest of components.

All experiments were performed at the ACC Cyfronet AGH-UST site on the SMP supercomputer, SGI Altix 3700, equipped with $1281.5 \mathrm{GHz}$ Intel Itanium 2 processors, $256 \mathrm{~GB}$ RAM and $4.75 \mathrm{~TB}$ disk storage. The taggers have been compiled with standard optimization level. Depending on baseline tagger chosen training time varies considerably from 3 to $980 \cdot 10^{2}$ seconds and tagging speed from 5 to $220 \cdot 10^{2}$ tokens/second [4]. 


\section{Results}

\subsection{Results for Baseline Methods}

The accuracy of 5 baseline tagging algorithms is presented in Table 4

Table 4. Accuracy of baseline taggers trained on $90 \%$ of the m-FDCP corpus, [\%]

\begin{tabular}{|l|c|c|c|c|c|c|}
\hline & TnT & MXP & fnTBL & MBT & SVM \\
\hline & \multicolumn{5}{|c|}{ Simple tagset } \\
\hline All tokens & 96.20 & 96.30 & 96.51 & 95.74 & 96.74 \\
Known tokens & 96.98 & 97.01 & 97.51 & 97.10 & 97.52 \\
Unknown tokens & 88.65 & 89.43 & 86.89 & 82.60 & 89.14 \\
Ambiguous tokens & 89.50 & 91.09 & 91.36 & 89.94 & 91.36 \\
Word segments & 95.46 & 95.57 & 95.83 & 94.91 & 96.10 \\
Word segments with known tags & 96.94 & 96.79 & 97.55 & 97.08 & 97.60 \\
Word segments with unknown tags & 0.00 & 28.21 & 3.85 & 0.00 & 0.00 \\
Unknown word segments & 88.65 & 89.43 & 86.90 & 82.60 & 89.15 \\
Sentences & 61.48 & 62.15 & 63.71 & 58.54 & 65.16 \\
\hline & \multicolumn{5}{|c|}{ Complex tagset } \\
\hline All tokens & 86.33 & 85.00 & 86.79 & 82.31 & 73.54 \\
Known tokens & 88.97 & 87.53 & 89.76 & 85.75 & 81.12 \\
Unknown tokens & 60.86 & 60.55 & 58.09 & 49.06 & 0.23 \\
Ambiguous tokens & 78.66 & 78.71 & 80.34 & 72.48 & 63.44 \\
Word segments & 83.66 & 82.07 & 84.21 & 78.85 & 68.36 \\
Word segments with known tags & 90.73 & 87.44 & 90.27 & 86.58 & 80.69 \\
Word segments with unknown tags & 0.00 & 29.84 & 30.50 & 0.66 & 0.00 \\
Unknown word segments & 60.86 & 60.55 & 58.09 & 49.05 & 0.23 \\
Sentences & 28.95 & 26.88 & 29.87 & 22.51 & 12.04 \\
\hline
\end{tabular}

\subsection{Results for Split Models}

The observed decline of accuracy with the thematic split model (Table 5 , columns $2-7$ ) is due to considerable reduction of training sets sizes, comparing to setup for the baseline algorithms, what might mask the profits of thematic split. To prevent the masking effect we made another experiment. We examined the baseline algorithms, with uniform (balanced) training and test set of identical sizes as for the thematic split, i.e., standing for $18 \%$ and $2 \%$ of the entire corpus (Table 5. column 8). The higher accuracy of the thematic split model over this additional experiment is apparent (column 8 vs. column 7 of Table 5 ).

We remark also accuracy degradation of the attribute split model (Table 6) comparing to the baseline taggers. Only the attribute split model for SVM experiences improvement, in comparison to the baseline tagger. This can be explained by considerably low accuracy of the SVM tagger. 
Table 5. Accuracy of taggers trained on thematic parts A-E, average accuracy (arithmetic mean) of all thematic parts, accuracy of taggers trained on the uniform set, $[\%]$

\begin{tabular}{|l|ccccc|c|c|}
\hline & T & B & C & D & E & average & $18 \% / 2 \%$ \\
uniform \\
\hline & \multicolumn{7}{|c|}{ Simple tagset } \\
\hline TnT & 95.24 & 96.49 & 95.39 & 95.07 & 95.24 & 95.49 & 94.82 \\
MXP & 94.63 & 95.98 & 94.66 & 94.83 & 94.85 & 94.99 & 94.46 \\
fnTBL & 94.98 & 95.63 & 95.20 & 94.58 & 95.40 & 95.16 & 94.64 \\
MBT & 94.14 & 95.61 & 94.40 & 94.00 & 94.69 & 94.57 & 93.80 \\
SVM & 95.44 & 96.62 & 95.49 & 95.39 & 95.83 & 95.75 & 94.93 \\
\hline & \multicolumn{7}{|c|}{ Complex tagset } \\
\hline TnT & 82.61 & 83.35 & 82.24 & 82.39 & 85.79 & 83.28 & 83.26 \\
MXP & 79.05 & 80.34 & 79.47 & 79.83 & 84.02 & 80.54 & 79.22 \\
fnTBL & 80.74 & 80.80 & 80.43 & 80.99 & 84.00 & 81.39 & 80.92 \\
MBT & 77.91 & 77.80 & 77.13 & 78.42 & 82.92 & 78.84 & 78.03 \\
SVM & 66.69 & 66.72 & 65.42 & 67.94 & 74.53 & 68.26 & 67.69 \\
\hline
\end{tabular}

Table 6. Tagging accuracy of the attribute split model applied to the complex tagset, in next rows accuracy of its individual components given, [\%]

\begin{tabular}{|l|c|c|c|c|c|}
\hline & TnT & MXP & fnTBL & MBT & SVM \\
\hline attribute split & 81.73 & 82.55 & 81.73 & 81.00 & 84.06 \\
\hline POS & 96.20 & 96.30 & 96.44 & 95.74 & 96.74 \\
number & 97.08 & 97.10 & 96.74 & 96.77 & 97.42 \\
case & 91.00 & 92.81 & 92.08 & 91.03 & 92.81 \\
gender & 92.46 & 91.95 & 91.13 & 91.86 & 93.01 \\
person & 99.31 & 99.45 & 99.54 & 99.38 & 99.53 \\
degree & 98.13 & 98.44 & 98.59 & 98.11 & 98.58 \\
aspect & 97.73 & 97.91 & 98.03 & 97.54 & 98.19 \\
negation & 98.82 & 98.91 & 98.91 & 98.70 & 98.92 \\
vocalicity & 100.00 & 100.00 & 100.00 & 100.00 & 100.00 \\
\hline
\end{tabular}

\subsection{Results for Voting Methods}

The possibility of improving the baseline algorithms by voting methods is expressed by complementarity (Table 7). Complementarity value near $0 \%$ indicates that a pair of taggers gives similar effects and no accuracy increase with voting methods. The closer the complementarity values to $100 \%$, the higher the margin for accuracy increase of voting methods.

The accuracy of the voting methods themselves is given in Table 8 . The methods are ordered according to their growing complexity. The effort connected with gathering weights required by voting methods and creation of the tuning set paid off. Voting methods achieve the highest accuracy among all presented algorithms. The results of Total Precision methods are especially encouraging. 
Table 7. Complementarity, $\operatorname{comp}(B \mid A)$, of baseline taggers trained on $90 \%$ of the m-FDCP corpus, [\%]

(a) simple tagset

\begin{tabular}{|l|c|c|c|c|c|}
\hline A & TnT & MXP & fnTBL & MBT & SVM \\
\hline TnT & - & 35.72 & 31.29 & 39.21 & 25.74 \\
MXP & 37.32 & - & 36.72 & 46.58 & 30.30 \\
fnTBL & 36.84 & 40.35 & - & 41.03 & 25.65 \\
MBT & 31.80 & 38.55 & 28.03 & - & 23.79 \\
SVM & 36.16 & 38.55 & 30.47 & 41.60 & - \\
\hline
\end{tabular}

(b) complex tagset

\begin{tabular}{|l|c|c|c|c|c|}
\hline A & TnT & MXP & fnTBL & MBT & SVM \\
\hline TnT & - & 39.13 & 32.28 & 39.25 & 55.39 \\
MXP & 33.20 & - & 33.25 & 43.19 & 56.42 \\
fnTBL & 34.56 & 41.22 & - & 41.07 & 54.05 \\
MBT & 21.39 & 33.01 & 21.09 & - & 39.96 \\
SVM & 13.62 & 23.12 & 7.94 & 10.17 & - \\
\hline
\end{tabular}

Table 8. Accuracy of voting methods, [\%]

\begin{tabular}{|l|c|c|}
\hline Voting method & Simple tagset & Complex tagset \\
\hline Majority & 96.93 & 87.14 \\
Total Precision & 96.95 & 88.03 \\
Tag Precision & 96.93 & 87.41 \\
Precision Recall & 96.93 & 87.21 \\
\hline
\end{tabular}

\section{Conclusions}

According to our experiments, the following conclusions can be drawn.

The SVM tagger achieves the highest, state-of-the-art accuracy among the baseline methods for the simple tagset, although is useless when applied to the complex tagset. For the complex tagset fnTBL yields both the highest overall accuracy and sentence accuracy among the baseline methods. If the amount of unknown tokens is prevailing, the MXPost should be considered.

Attribute split models give lower results than the baseline taggers except the SVM case, whose baseline accuracy is however significantly low.

The thematic split model has not outperformed any baseline tagger. The additional experiment with uniform $20 \%$ set of the m-FDCP corpus proved however potential usefulness of the model. The condition of its applicability is that a corpus consists of several thematic segments. The segments should be large enough, so that the benefit of separate thematic models dominates accuracy degradation tied to reduction of a training set size.

Voting methods are the most complex methods, requiring preparation of several baseline taggers. Elaboration of a reliable tuning set is another computationally demanding issue. Each of these methods outperforms the most accurate baseline method. The Total Precision method achieves the highest accuracy and at the same time is simpler than the Tag Precision and Precision Recall methods yielding the simplicity only to the Majority method.

Acknowledgments. This research is partially supported by the Polish Ministry of Science and Higher Education, grant no. 11.11.120.777. ACC CYFRONET AGH is acknowledged for the computing time. 


\section{References}

1. Mauco, M., Leonardi, M.: A derivation strategy for formal specifications from natural language requirements models. Computing and Informatics 26(4), 421-445 (2007)

2. Kuta, M., Chrząszcz, P., Kitowski, J.: Increasing quality of the Corpus of Frequency Dictionary of Contemporary Polish for morphosyntactic tagging of the Polish language. Computing and Informatics (to appear)

3. Corpus of Frequency Dictionary of Contemporary Polish, http://www.mimuw.edu.pl/polszczyzna

4. Kuta, M., Chrząszcz, P., Kitowski, J.: A case study of algorithms for morphosyntactic tagging of Polish language. Computing and Informatics 26(6), 627-647 (2007)

5. Ratnaparkhi, A.: A maximum entropy model for part-of-speech tagging. In: Proc. of the 1st Conf. on Empirical Methods in Natural Language Processing, Univ. of Pennsylvania, USA, pp. 133-142 (1996)

6. Daelemans, W., Zavrel, J., Berck, P., Gillis, S.: MBT: A memory-based part of speech tagger-generator. In: Proc. of the 4th Workshop on Very Large Corpora, Copenhagen, Denmark, pp. 14-27 (1996)

7. Brill, E.: Transformation-based error-driven learning and natural language processing: A case study in part of speech tagging. Computational Linguistics 21(4), 543-565 (1995)

8. Giménez, J., Màrquez, L.: SVMTool: A general POS tagger generator based on Support Vector Machines. In: Proc. of the 4th Int. Conf. on Language Resources and Evaluation, Lisbon, Portugal, pp. 43-46 (2004)

9. Brants, T.: TnT - a statistical part-of-speech tagger. In: Proc. of the 6th Applied Natural Language Processing Conf., Seattle, USA, pp. 224-231 (2000)

10. Florian, R., Ngai, G.: Fast Transformation-Based Learning Toolkit manual. John Hopkins Univ., USA (2001), http://nlp.cs.jhu.edu/〜rflorian/fntbl

11. van Halteren, H., Zavrel, J., Daelemans, W.: Improving accuracy in word class tagging through the combination of machine learning systems. Computational Linguistics 27(2), 199-229 (2001)

12. van Halteren, H., Zavrel, J., Daelemans, W.: Improving data driven wordclass tagging by system combination. In: Proc. of the 36th Annual Meeting on Association for Computational Linguistics, Montréal, Canada, vol. 1, pp. 491-497 (1998)

13. Schröder, I.: A Case Study in Part-of-Speech Tagging Using the ICOPOST Toolkit, Technical report FBI-HH-M-314/02, Univ. of Hamburg, Germany (2002)

14. Brill, E., Wu, J.: Classifier combination for improved lexical disambiguation. In: Proc. of the 7th Int. Conf. on Computational Linguistics, San Francisco, USA, pp. 191-195 (1998)

15. Modified corpus of Frequency Dictionary of Contemporary Polish, http://nlp.icsr.agh.edu.pl

16. IPI PAN Corpus resources, http://korpus.pl 